\title{
Single-channel correlators and residue calculus
}

\author{
P. Jacob and P. Mathieu \\ Département de physique, \\ Université Laval, \\ Québec, Canada G1K 7P4 \\ (pjacob@phy.ulaval.ca, pmathieu@phy.ulaval.ca)
}

\begin{abstract}
Some simple (namely, single-channel) correlation functions involving an arbitrary number of fields are computed by means of a direct application of the residue calculus, through partial fraction expansions. Examples are presented in minimal models and parafermionic conformal theories. A generic factorized expression is deduced for the corresponding singlechannel structure constants.
\end{abstract}

$05 / 01$ 


\section{Introduction}

\subsection{Correlators, OPEs and Ward identities}

The plain method for evaluating $N$-point correlation functions, given the OPEs (operator product expansions) of the fields whose correlations are to be computed, amounts to substitute the OPE of two fields in order to reduce the correlator to $(N-1)$-point functions and iterate this procedure until the result becomes expressed in terms of a threepoint function. The later being known exactly up to structure constants, the correlator is then expressed in terms of these constants. However, the reduction in the number of points has been traded for a new complication: we then have to sum up the infinite series (i.e., conformal blocks) associated to each OPE. In principle, these can be summed exactly only in simple cases. A sample computation is presented in Appendix A.

For the mere formulation of the model, the correlators that are particularly important are those involving the symmetry generators, namely the generators of the extended conformal algebra. Their relevance lie in that the internal coherence of the extended algebra boils down to a precise statement concerning the correlators of the symmetry generators: all their four-point functions must be associative. The associativity requirement is the condition that a correlation function can be calculated in many different ways, in particular, by evaluating the OPEs in different orders, without affecting the result. (This is the way the structure constants are calculated.) But testing associativity calls for the exact form of these correlation functions.

However, in this particular instance, the problem appears to be tractable. Indeed, the evaluation of correlation functions involving extended conformal-algebra generators is usually rather simple in that only the singular terms have to be considered. Take for instance a correlation function involving the energy-momentum tensor $T(z)$ and some

primary fields $\prod_{j=1}^{N} \phi_{j}\left(z_{j}\right)$. This is certainly relevant to the question of studying the associativity of a given conformal algebra because the algebra generators other that $T$ have to be Virasoro primary fields. It is a very basic fact that in eliminating $T(z)$ through OPE, one simply needs to take into account the singular terms in the product of $T(z)$ with all the other fields in the correlator:

$$
\left\langle T(z) \prod_{j=1}^{N} \phi_{j}\left(z_{j}\right)\right\rangle=\sum_{i=1}^{N}\left\{\frac{h_{i}}{\left(z-z_{i}\right)^{2}}+\frac{\partial_{z_{i}}}{z-z_{i}}\right\}\left\langle\prod_{j=1}^{N} \phi_{j}\left(z_{j}\right)\right\rangle
$$

In other words, in this special case we do not have to keep track of an infinite series. This is a consequence of the conformal Ward identities - cf. [1, 2, 3]. 


\subsection{Correlators involving $T$ as meromorphic functions}

But if we think about this result from the point of view of OPEs, it looks rather surprising that by considering only the singular terms of the OPE of $T$ with the other fields of the correlator (and not the complete infinite OPE series) we can compute the correlation function exactly. This has a natural complex-analysis explanation: the result simply corresponds to the partial fraction expansion of the meromorphic function representing the correlation, viewed as a function of one of its field. For instance, the correlator in (1.1), considered as a function of $z$, is a meromorphic function with double poles at the various $z_{j}$ (since the $\phi_{j}\left(z_{j}\right)$ 's are supposed to be primary) with coefficients fixed by the OPE $T(z) \phi_{j}\left(z_{j}\right)$. In that case, the partial fraction expansion is complete in that there is no additional analytic piece. Indeed, this meromorphic function vanishes at infinity since $T(z) \sim z^{-4}$ as $z \rightarrow \infty$. Such a function is simply given by the sum of the principal parts at the various poles.

Let us make the above statements more explicit. Recall that a meromorphic function with vanishing analytic part can be written in partial fraction as

$$
F(z)=\sum_{j=1}^{N} \sum_{r=1}^{n_{j}} \frac{a_{r}^{(j)}}{\left(z-z_{j}\right)^{r}}
$$

where $n_{j}$ is the order of the pole at $z_{j}$ and the coefficients $a_{r}^{(j)}$ are given by

$$
a_{n_{j}-k}^{(j)}=\lim _{z \rightarrow z_{j}} \frac{1}{k !} \frac{\partial^{k}}{\partial z^{k}}\left(z-z_{j}\right)^{n_{j}} F(z)
$$

When $F(z)$ is a correlation function, the various coefficients $a_{r}^{(j)}$ are expressed in terms of lower-order correlation functions. On the other hand, if the meromrophic function $F(z)$ does not vanish at infinity, an analytic function needs to be added to this sum of principal parts. Suppose that $F(z)$ behaves rather like $F(z) \sim z^{p}$ as $z \rightarrow \infty$, with $p$ integer. This signals the presence of a pole of order $p$ at infinity so that the principal part at infinity (in which we include a constant term) has to be taken into account. The expression of $F(z)$ becomes then

$$
F(z)=\sum_{j=1}^{N} \sum_{r=1}^{n_{j}} \frac{a_{r}^{(j)}}{\left(z-z_{j}\right)^{r}}+\sum_{k=1}^{p} a_{k}^{(\infty)} z^{k}
$$

The last sum is the analytic part of $F(z)$. 
It is then completely obvious that in the case (1.1), the principal part at $z_{j}$ is nothing but the sum of the singular terms in the $\mathrm{OPE} T(z) \phi_{j}\left(z_{j}\right)$. Indeed, viewing the correlator as a function of $z$, we have:

$$
\left\langle T(z) \prod_{j=1}^{N} \phi_{j}\left(z_{j}\right)\right\rangle=\sum_{i=1}^{N}\left(\frac{a_{2}^{(i)}}{\left(z-z_{i}\right)^{2}}+\frac{a_{1}^{(i)}}{\left(z-z_{i}\right)}\right)
$$

with

$$
\begin{aligned}
a_{2}^{(i)} & =\lim _{z \rightarrow z_{i}}\left(z-z_{i}\right)^{2}\left\langle T(z) \prod_{j=1}^{N} \phi_{j}\left(z_{j}\right)\right\rangle \\
& =\lim _{z \rightarrow z_{i}}\left(z-z_{i}\right)^{2}\left\langle\prod_{j=1}^{i-1} \phi_{j}\left(z_{j}\right)\left(\frac{h_{i} \phi_{i}\left(z_{i}\right)}{\left(z-z_{i}\right)^{2}}+\frac{\partial_{z_{i}} \phi_{i}\left(z_{i}\right)}{z-z_{i}}+\cdots\right) \prod_{j=i+1}^{N} \phi_{j}\left(z_{j}\right)\right\rangle \\
& =h_{i}\left\langle\prod_{j=1}^{N} \phi_{j}\left(z_{j}\right)\right\rangle
\end{aligned}
$$

and

$$
\begin{aligned}
a_{1}^{(i)} & =\lim _{z \rightarrow z_{i}} \frac{\partial}{\partial z}\left(z-z_{i}\right)^{2}\left\langle T(z) \prod_{j=1}^{N} \phi_{j}\left(z_{j}\right)\right\rangle \\
& =\lim _{z \rightarrow z_{i}} \frac{\partial}{\partial z}\left\{\left\langle h_{i} \prod_{j=1}^{N} \phi_{j}\left(z_{j}\right)\right\rangle+\left(z-z_{i}\right) \partial_{i}\left\langle\prod_{j=1}^{N} \phi_{j}\left(z_{j}\right)\right\rangle+\cdots\right\} \\
& =\partial_{i}\left\langle\prod_{j=1}^{N} \phi_{j}\left(z_{j}\right)\right\rangle
\end{aligned}
$$

(the first term of the second line drops because it is independent of $z$ ); this gives the rhs of (1.1).

The above derivation of (1.1) makes clear the rather auxiliary aspect of the primary nature of the fields inside the correlator. For instance, one could introduce quasi-primary 
fields, e.g., $T(z)$ itself. Regarding the following correlator as a function of $\zeta_{1}$ leads to [2]:

$$
\begin{aligned}
& \left\langle\prod_{i=1}^{M} T\left(\zeta_{i}\right) \prod_{j=1}^{N} \phi_{j}\left(z_{j}\right)\right\rangle \\
& \quad=\sum_{i=2}^{M} \frac{c / 2}{\left(\zeta_{1}-\zeta_{i}\right)^{4}}\left\langle\prod_{\substack{k=2 \\
k \neq i}}^{M} T\left(\zeta_{k}\right) \prod_{j=1}^{N} \phi_{j}\left(z_{j}\right)\right\rangle \\
& \quad+\sum_{i=2}^{M}\left(\frac{2}{\left(\zeta_{1}-\zeta_{i}\right)^{2}}+\frac{1}{\zeta_{1}-\zeta_{i}} \frac{\partial}{\partial \zeta_{i}}\right)\left\langle\prod_{k=2}^{M} T\left(\zeta_{k}\right) \prod_{j=1}^{N} \phi_{j}\left(z_{j}\right)\right\rangle \\
& \quad+\sum_{i=1}^{N}\left(\frac{1}{\left(h_{i}-z_{i}\right)^{2}}+\frac{\partial}{\zeta_{1}-z_{i}} \frac{\partial}{\partial z_{i}}\right)\left\langle\prod_{k=2}^{M} T\left(\zeta_{k}\right) \prod_{j=1}^{N} \phi_{j}\left(z_{j}\right)\right\rangle
\end{aligned}
$$

A manifestation of the associativity property is that the same expression for this correlator follows by considering it as a meromorphic function of another variable $\zeta_{i}$.

Even 'less-primary' fields are treated by the same method: only the singular terms of the OPE with $T(z)$ contribute.

\subsection{Generalizing the class of correlators computable from partial fraction expansions}

What is central in the previous computations of correlators involving $T$ ? At first, there is the fact that the OPE of $T$ with another field has only one channel, i.e., all those terms that appear in the $\mathrm{OPE} T(z) \phi(w)$ belong to the conformal family of $\phi(w)$. This ensures that the different powers of $z-z_{j}$ (for a given $j$ ) all differ by integers. A second important point is that $T$ is local with respect to any other field in the theory, which implies that, not only the different powers of $z-z_{j}$ differ by integers, but they are all integers themselves. In other words, the $\mathrm{OPE} T(z) \phi(w)$ is a genuine Laurent series.

Therefore, this computation method can be used for calculating the different four-point functions for the extended-algebra generators when these generators $\mathcal{Y}_{i}$ all have integer dimensions. Note that in this case, the one-channel constraint is superfluous because all the powers of $z-w$ in the $\mathrm{OPE} \mathcal{Y}_{i}(z) \mathcal{Y}_{j}(w) \in \sum_{k}\left[\mathcal{Y}_{k}(w)\right]$ have automatically integer dimension. A well-known example of this type is the WZW model whose extended symmetry is an affine Lie algebra, with generators $J^{a}$ satisfying the OPE $J^{a} \times J^{b}=\mathbb{I}+J^{c}$.

But if this is to be used for the analysis of more general extended algebras, for instance parafermionic models with fields having fractional dimension, one has to be able to tackle 
situations where there are branching points, that is, when the powers of $z-w$ are fractional in the $\mathrm{OPE} \mathcal{Y}_{i}(z) \mathcal{Y}_{j}(w)$. The cure is quite simple: we just have to modify the correlation function, which is viewed as a function of the first variable $z_{1}$ - the position of a given symmetry generator, say $\mathcal{Y}\left(z_{1}\right)$-, by multiplying it by appropriate powers of $z_{1}-z_{j}\left(z_{j}\right.$ being the position of another field inside of the correlator) in order to transform it into a meromorphic function of $z_{1}$ [4]. In other words, to analyze the correlation $\left\langle\mathcal{Y}\left(z_{1}\right) \cdots\right\rangle$, we consider the intermediate function

$$
F\left(z_{1}\right)=\left(\prod_{j \geq 2}\left(z_{1 j}\right)^{d_{j}}\right)\left\langle\mathcal{Y}\left(z_{1}\right) \cdots\right\rangle
$$

for those values of $d_{i}$ appropriate to make $F\left(z_{1}\right)$ meromorphic. The OPEs fix then the position of the poles of this function together with their residues. This determines $F\left(z_{1}\right)$ and, thereby, the correlation function under consideration. Notice however that in the present case, the principal part of $F\left(z_{1}\right)$ at $z_{j}$ is not given solely by the sum of the singular terms in $z_{1}-z_{j}$ : for the subleading terms in the principal series, there are derivatives in the expression for the constants $a_{n_{j}-k}^{(j)}$ in (1.3) and these derivatives do not select exclusively the corresponding singular terms due to the presence of the prefactors $\prod_{i \geq 2}\left(z_{1 i}\right)^{d_{i}}$ on which the derivatives also act.

As already pointed out, the presence or absence of a regular (analytic) part in the expression of $F\left(z_{1}\right)$ is fixed by the behavior of the field at position $z_{1}$, as $z_{1} \rightarrow \infty$, e.g., $\mathcal{Y}\left(z_{1}\right) \sim z_{1}^{-2 h \mathcal{Y}}$, together with the prefactor composed of the different fractional powers of $z_{1}-z_{j}$ that have been introduced. A modification of the prefactor by an integer power of $z_{1 j}$ obviously affects the nature of the regular part of $F\left(z_{1}\right)$ but not the final expression of the correlator.

By bringing out the conditions underlying the applicability of the residue method to the computation of correlators involving $T$, we have identified at once two criteria: (1) the single-channel requirement and (2) the locality condition. The simple trick just described for transforming a function with branching singularities into a meromorphic function provides thus a way of bypassing the apparent second limitation.

In relation with the single-channel requirement, we have already presented a situation in which it can be relaxed, namely for correlators of symmetry generators all having integer dimension. Phrased in more general terms, the one-channel condition is not mandatory 
when the OPEs of the fields inside the correlators close in a set of fields which all have conformal dimension that differs from each other by integers. But this is a rather special instance. In more general circumstances, the single-channel requirement cannot be avoided. Notice however that this is not as restricting as it looks at first sight. What is really needed is a not exactly a genuine single-channel OPE but, rather, a correlation function that selects a single channel in each intermediate OPE.

In the above considerations there is in addition an implicit third limitation, which is that the field in terms of which the partial fraction is formulated has to be a symmetry generator. But the complex analysis is blind to the subtle conformal nature of the field evaluated at $z_{1}$. It is clear that it can be any field, as long as (generically) the correlation function has effectively a single channel. In particular, it can be applied to the calculation of special correlators involving only primary fields. Examples of such functions containing only Virasoro or parafermionic primary fields are presented below.

\section{Correlators of Virasoro degenerate primary fields}

We will first consider the following correlation function of Virasoro degenerate primary fields:

$$
\left\langle\phi_{12}\left(z_{1}\right) \cdots \phi_{12}\left(z_{n}\right) \phi_{1, n+1}\left(z_{n+1}\right)\right\rangle
$$

Given the fusion rule [1]

$$
\phi_{12} \times \phi_{1 r}=\phi_{1, r-1}+\phi_{1, r+1}
$$

we see that the insertion of the field $\phi_{1, n+1}$ in the last position effectively selects a single channel, i.e., a single term contributes from each OPE. This is most easily seen for the case $n=2$ corresponding to the three-point function: substituting $\phi_{12} \times \phi_{12}=\phi_{11}+\phi_{13}$ in the correlator and using the orthogonality condition

$$
\left\langle\phi_{1 r}\left(z_{1}\right) \phi_{1 s}\left(z_{2}\right)\right\rangle=\delta_{r, s}
$$

it is clear that it is only the $\phi_{13}$ term does contribute. 
Recall that $\phi_{r s}$, with $r, s$ integers, refers to a Virasoro primary field that contains a singular descendant (hence the qualitative 'degenerate') at level $r s$ and whose dimension $h_{r s}$ is linked to the central charge via a parameter $t$ as follows:

$$
\begin{aligned}
h_{r s}(t) & =\frac{1}{4}\left(r^{2}-1\right) t+\frac{1}{4}\left(s^{2}-1\right) \frac{1}{t}-\frac{1}{2}(r s-1) \\
c(t) & =13-6\left(t+\frac{1}{t}\right)
\end{aligned}
$$

It is convenient to set

$$
a=\frac{1}{2 t}
$$

In this notation, the dimension $h_{1 n}$ takes the form

$$
h_{1 n}=\frac{1}{2}\left(n^{2}-1\right) a-\frac{1}{2}(n-1)
$$

so that the power of $(z-w)$ of the leading term of the family $\phi_{1 m}$ in the OPE of $\phi_{1 r} \times \phi_{1 s}$ is

$$
h_{1 r}+h_{1 s}-h_{1 m}=\frac{1}{2}\left(r^{2}+s^{2}-m^{2}-1\right) a-\frac{1}{2}(r+s-m-1)
$$

In order to lighten further the notation, we will set

$$
\phi_{1, r+1} \equiv \mathcal{P}_{r}
$$

and define the structure constants $c_{r s}$ as follows

$$
\mathcal{P}_{r}(z) \mathcal{P}_{s}(w) \sim c_{r s} \mathcal{P}_{r+s}+\cdots
$$

Therefore, in terms of the minimal-model structure constants, the $c_{r s}$ are

$$
c_{r s}=C_{(1, r+1),(1, s+1)}{ }^{(1, r+s+1)}=C_{(1, r+1),(1, s+1),(1, r+s+1)}
$$

the last expression being symmetric with respect to the interchange of any two pairs of the three indices.

Let us evaluate the four-point function

$$
\left\langle\phi_{12}\left(z_{1}\right) \phi_{12}\left(z_{2}\right) \phi_{12}\left(z_{3}\right) \phi_{14}\left(z_{4}\right)\right\rangle=\left\langle\mathcal{P}_{1}\left(z_{1}\right) \mathcal{P}_{1}\left(z_{2}\right) \mathcal{P}_{1}\left(z_{3}\right) \mathcal{P}_{3}\left(z_{4}\right)\right\rangle
$$

by the residue method. The following function

$$
F\left(z_{1}\right)=z_{12}^{-a} z_{13}^{-a} z_{14}^{5 a-1}\left\langle\mathcal{P}_{1}\left(z_{1}\right) \mathcal{P}_{1}\left(z_{2}\right) \mathcal{P}_{1}\left(z_{3}\right) \mathcal{P}_{3}\left(z_{4}\right)\right\rangle
$$


turns out to an analytic function of $z_{1}$ (there are no poles). Actually, it is simply a constant, as the behavior as $z_{1} \rightarrow \infty$ indicates, i.e.,

$$
F\left(z_{1}\right) \sim z_{1}^{-a} z_{1}^{-a} z_{1}^{5 a-1} \frac{1}{z_{1}^{3 a-1}} \quad\left(z_{1} \rightarrow \infty\right)
$$

where the last term is the contribution of the correlator per se, in which only $\mathcal{P}_{1}\left(z_{1}\right)$ contributes: $\mathcal{P}_{1}\left(z_{1}\right) \sim z_{1}^{-2 h_{12}}$. This constant can be evaluated in many different ways and in particular, in the limit $z_{1} \rightarrow z_{4}$ :

$$
\begin{aligned}
\lim _{z_{1} \rightarrow z_{4}} F\left(z_{1}\right) & =\lim _{z_{1} \rightarrow z_{4}} z_{12}^{-a} z_{13}^{-a} z_{14}^{5 a-1} \frac{c_{11}}{z_{14}^{5 a-1}}\left\langle\mathcal{P}_{2}\left(z_{2}\right) \mathcal{P}_{1}\left(z_{3}\right) \mathcal{P}_{3}\left(z_{4}\right)\right\rangle \\
& =\lim _{z_{1} \rightarrow z_{4}} z_{12}^{-a} z_{13}^{-a} z_{14}^{5 a-1} \frac{c_{11}}{z_{14}^{5 a-1}} \frac{c_{12}}{z_{23}^{-a} z_{24}^{4 a-1} z_{34}^{4 a-1}} \\
& =\frac{c_{11} c_{12}}{z_{23}^{-a} z_{24}^{5 a-1} z_{34}^{5 a-1}}
\end{aligned}
$$

Therefore, we have

$$
\left\langle\mathcal{P}_{1}\left(z_{1}\right) \mathcal{P}_{1}\left(z_{2}\right) \mathcal{P}_{1}\left(z_{3}\right) \mathcal{P}_{3}\left(z_{4}\right)\right\rangle=\frac{c_{11} c_{12}}{z_{12}^{-a} z_{13}^{-a} z_{23}^{-a} z_{14}^{5 a-1} z_{24}^{5 a-1} z_{34}^{5 a-1}}
$$

This computation can be easily generalized to the case where there is an arbitrary number of $\mathcal{P}_{1}$ fields projected onto an appropriate $\mathcal{P}_{n}$ field enforcing the single-channel constraint:

$$
\left\langle\mathcal{P}_{1}\left(z_{1}\right) \cdots \mathcal{P}_{1}\left(z_{n}\right) \mathcal{P}_{n}\left(z_{n+1}\right)\right\rangle=c_{11} c_{12} \cdots c_{1, n-1} \prod_{1 \leq i<j \leq n} \frac{1}{z_{i j}^{-a}} \prod_{1 \leq \ell \leq n} \frac{1}{z_{\ell, n+1}^{(n+2) a-1}}
$$

It is not difficult to verify that this correlator is solution of the $\phi_{12}$ singular-vector differential equation. Having computed our correlator without resorting to this differential equation, one could ask where does the singular nature of $\phi_{12}$ enters, if it does at all. It is actually used right at the beginning, in specifying the fusion rules.

Still using the residue method, we can derive the following other generalization of the four-point function (2.15):

$$
\begin{aligned}
\left\langle\mathcal{P}_{1}\left(z_{1}\right) \mathcal{P}_{n}\left(z_{2}\right)\right. & \left.\mathcal{P}_{n^{\prime}}\left(z_{3}\right) \mathcal{P}_{n+n^{\prime}+1}\left(z_{4}\right)\right\rangle \\
& =\frac{c_{1, n+n^{\prime}} c_{n, n^{\prime}}}{z_{12}^{-n a} z_{13}^{-n^{\prime} a} z_{14}^{\left(3+n+n^{\prime}\right) a-1} z_{23}^{-n n^{\prime} a} z_{24}^{\left(n^{2}+3 n+n n^{\prime}\right) a-n} z_{34}^{\left(n^{\prime 2}+3 n^{\prime}+n n^{\prime}\right) a-n^{\prime}}}
\end{aligned}
$$


A different ordering in the evaluation of the constant representing the intermediate meromorphic function forces the relation

$$
c_{1, n+n^{\prime}} c_{n, n^{\prime}}=c_{1, n} c_{n+1, n^{\prime}}
$$

The solution of this recursion relation reads

$$
c_{n, n^{\prime}}=\frac{c_{1,1} \cdots c_{1, n+n^{\prime}-1}}{c_{1,1} \cdots c_{1, n-1} c_{1,1} \cdots c_{1, n^{\prime}-1}}
$$

which, as it should, is symmetric in both indices.

We now want to stress that the factorized expression (2.18) was actually coded in the correlator (2.16). This correlator was computed by contracting all the $\mathcal{P}_{1}$ fields from left to right. Equivalently, we could have stop this process at the $m$-th one and contract the remaining $\mathcal{P}_{1}$ 's from right to left up to the $m^{\prime}$-th. That yields (with $n=m+m^{\prime}$ ):

$$
\begin{gathered}
{\left[c_{11} c_{12} \cdots c_{1, m-1}\right]\left[c_{11} c_{12} \cdots c_{1, m^{\prime}-1}\right]\left\langle\mathcal{P}_{m} \mathcal{P}_{m^{\prime}} \mathcal{P}_{m+m^{\prime}}\right\rangle} \\
\sim\left[c_{11} c_{12} \cdots c_{1, m-1}\right]\left[c_{11} c_{12} \cdots c_{1, m^{\prime}-1}\right] c_{m, m^{\prime}}
\end{gathered}
$$

The comparison between the two results yields directly (2.19).

Equation (2.19) shows that all the constants $c_{n, n^{\prime}}$ can be calculated in terms of the $c_{1, n}$ 's only.1 In order to calculate the structure constants $c_{1, n}$, we need to evaluate the four-point function $\left\langle\mathcal{P}_{1} \mathcal{P}_{n} \mathcal{P}_{1} \mathcal{P}_{n}\right\rangle$; but in this case, the two channels in the OPE $\mathcal{P}_{1} \times \mathcal{P}_{n}$ do contribute, which invalidates the applicability of the residue method. This correlator can be evaluated by using the singular-vector equations [1] or by using screening operators $[5,6,7]$. But this will not be reconsidered here. Our main point was to unravel the factorization (2.19), as well as illustrating the residue method.

We can similarly write down rather directly the expression for all correlators of the form

$$
\begin{aligned}
& \left\langle\mathcal{P}_{r_{1}}\left(z_{1}\right) \mathcal{P}_{r_{2}}\left(z_{2}\right) \cdots \mathcal{P}_{r_{n}}\left(z_{n}\right) \mathcal{P}_{R}\left(z_{n+1}\right)\right\rangle \\
& =c_{s_{1}, r_{2}} c_{s_{2}, r_{3}} \cdots c_{s_{n-1}, r_{n}} \prod_{1 \leq i<j \leq n} \frac{1}{z_{i j}^{-r_{i} r_{j} a}} \prod_{1 \leq \ell \leq n} \frac{1}{z_{\ell, n+1}^{r_{\ell}[(R+2) a-1]}}
\end{aligned}
$$

1 We stress that the $c_{n, n^{\prime}}$ 's form a particular class of structure constants, first in that they pertain to the restricted $\left\{\phi_{1, r}\right\}$ algebra and second, because they are those in front of the 'highest field', i.e., the maximal value of $m$, in the OPE $\mathcal{P}_{r} \times \mathcal{P}_{s} \sim \sum_{m} \mathcal{P}_{m}$. 
with $s_{i}=\sum_{j=1}^{i} r_{j}$ and $\sum_{i=1}^{n} r_{i}=R$.

Still more generally, we can also use the residue method to evaluate the correlators

$$
\left\langle\phi_{21}\left(z_{1}\right) \cdots \phi_{21}\left(z_{r-1}\right) \phi_{12}\left(z_{r}\right) \cdots \phi_{12}\left(z_{r+s-1}\right) \phi_{r s}\left(z_{r+s}\right)\right\rangle
$$

since they also involve a single channel. The corresponding structures constants satisfy

$$
C_{(r, s),\left(r^{\prime}, s^{\prime}\right)}{ }^{\left(r+r^{\prime}-1, s+s^{\prime}-1\right)}=C_{(r, 1),\left(r^{\prime}, 1\right)}{ }^{\left(r+r^{\prime}-1,1\right)} C_{(1, s),(1, s,)^{(1, s+s-1)}}=c_{r r^{\prime}} c_{s s^{\prime}}
$$

Are these results completely surprising? From the Coulomb-gas representation point of view, these expressions are somewhat trivial: these are the very correlators that do not require the insertion of even a single screening operator (and for this reason they have not been considered in $[5,6])$. A Coulomb-gas correlation function without screening is simply that of a collection of vertex operators, the result of which being quite simple and well-known (see e.g., eq. (9.9) of [3]). All the $z_{i j}$ dependence of the correlators is recovered in this way, the remaining factors being simply the structure constants.

However we stress that in our computation we do not require the free-field representation. In this sense the present derivation is thus more fundamental, in spite of the fact that it is applicable to a rather limited class of correlators. It also reveals a simple factorization of some of the structure constants that may not have been obvious from other points of view, but whose generality is by now quite transparent.

\section{Correlators in parafermionic models}

\subsection{Reviewing the $\mathbb{Z}_{k}$ parafermionic algebra}

The $\mathbb{Z}_{k}$ parafermionic conformal algebra $[4,8]$ (see also $[9,10]$ is generated by $k$ conserved holomorphic (and similar anti-holomorphic) parafermionic fields $\psi_{n}, n=$ $0,1, \cdots, k-1$, with $\psi_{0}=I$ and $\psi_{n}^{\dagger}=\psi_{k-n}$, with conformal dimension $h_{\psi_{n}}$ satisfying

$h_{\psi_{n}}=h_{\psi_{k-n}}$. The set of conformal dimensions $\left\{h_{\psi_{n}}\right\}$ is an input of a parafermionic theory and for the $\mathbb{Z}_{k}$ model, they read

$$
h_{\psi_{n}}=\frac{n(k-n)}{k}
$$


The $\psi_{n}$ are primary fields that form a closed algebra specified by the following OPEs:

$$
\begin{aligned}
\psi_{n}(z) \psi_{n^{\prime}}(w) & \sim \frac{\bar{c}_{n, n^{\prime}}}{(z-w)^{2 n n^{\prime} / k}} \psi_{n+n^{\prime}}(w) \quad\left(n+n^{\prime}<k\right) \\
\psi_{n}(z) \psi_{n^{\prime}}^{\dagger}(w) & \sim \frac{\bar{c}_{n, k-n^{\prime}}}{(z-w)^{2 \min \left(n, n^{\prime}\right)-2 n n^{\prime} / k}} \psi_{k+n-n^{\prime}}(w) \quad\left(n+n^{\prime}<k\right) \\
\psi_{n}(z) \psi_{n}^{\dagger}(w) & \sim \frac{1}{(z-w)^{2 n(k-n) / k}}\left[I+(z-w)^{2} \frac{2 h_{\psi_{n}}}{c} T(w)+\cdots\right]
\end{aligned}
$$

where the central charge is fixed by associativity to be

$$
c=\frac{2(k-1)}{(k+2)}
$$

The remaining OPE are obtained by conjugation and the condition

$$
\bar{c}_{n, n^{\prime}}=\bar{c}_{k-n, k-n^{\prime}}
$$

which implies, in particular, that $\bar{c}_{n, k-n^{\prime}}=\bar{c}_{k-n, n^{\prime}}$. The constants $\bar{c}_{n, n^{\prime}}$ are assumed to be real. They are fixed by the associativity conditions:

$$
c_{n, n^{\prime}}^{2}=\frac{\Gamma\left(n+n^{\prime}+1\right) \Gamma(k-n+1) \Gamma\left(k-n^{\prime}+1\right)}{\Gamma(n+1) \Gamma\left(n^{\prime}+1\right) \Gamma\left(k-n-n^{\prime}+1\right) \Gamma(k+1)} \quad\left(n+n^{\prime}<k\right)
$$

Note that this yields $\bar{c}_{n, k-n}=1$. They satisfy

$$
\bar{c}_{n, n^{\prime}}=\bar{c}_{n, k-n-n^{\prime}}=\bar{c}_{n^{\prime}, k-n-n^{\prime}}
$$

which reflects the symmetry of the three-point function:

$$
\left\langle\psi_{n}\left(z_{1}\right) \psi_{n^{\prime}}\left(z_{2}\right) \psi_{n+n^{\prime}}^{\dagger}\left(z_{3}\right)\right\rangle=\frac{C_{n, n^{\prime}, n+n^{\prime}}}{z_{12}^{2 n n^{\prime} / k} z_{23}^{2 n^{\prime}-2 n^{\prime}\left(n+n^{\prime}\right) / k} z_{13}^{2 n-2 n\left(n+n^{\prime}\right) / k}}
$$

\section{2. $\mathbb{Z}_{k}$ parafermionic correlators and their structure constants}

As a simple illustrative example of the application of the residue method to a parafermionic correlator, let us first rederive the well-known expression for the three-point correlation function

$$
\left\langle\psi_{1}\left(z_{1}\right) \psi_{1}\left(z_{2}\right) \psi_{2}^{\dagger}\left(z_{3}\right)\right\rangle
$$

Given the structure of the OPEs, we know that the function

$$
F\left(z_{1}\right)=z_{12}^{2 / k} z_{13}^{-4 / k}\left\langle\psi_{1}\left(z_{1}\right) \psi_{1}\left(z_{2}\right) \psi_{2}^{\dagger}\left(z_{3}\right)\right\rangle
$$


is a meromorphic function of $z_{1}$ with a double pole at $z_{3}$ and no regular terms. Therefore, this function has to be of the form:

$$
F\left(z_{1}\right)=\frac{a_{2}}{z_{13}^{2}}+\frac{a_{1}}{z_{13}}
$$

The first coefficient is

$$
a_{2}=\lim _{z_{1} \rightarrow z_{3}} z_{13}^{2} z_{12}^{2 / k} z_{13}^{-4 / k}\left\langle\psi_{1}\left(z_{1}\right) \psi_{1}\left(z_{2}\right) \psi_{2}^{\dagger}\left(z_{3}\right)\right\rangle
$$

Since we will be interested also in the second order term, we will need to consider also the subleading contribution in the limiting value of the correlation function. Recall that in the OPE of $\phi_{a}$ and $\phi_{b}$, the first two contributing terms in the conformal family of $\phi_{c}$ are

$$
\phi_{a}(z) \phi_{b}(w) \sim \frac{C_{a b c}}{(z-w)^{h_{a}+h_{b}-h_{c}}}\left[\phi_{c}(w)+\frac{\left(h_{a}-h_{b}+h_{c}\right)}{2 h_{c}}(z-w) \partial \phi_{c}(w)+\cdots\right]
$$

In the present case, we need

$$
\psi_{1}\left(z_{1}\right) \psi_{2}^{\dagger}\left(z_{3}\right) \sim \frac{\bar{c}_{1,1}}{z_{13}^{2-4 / k}}\left[\psi_{1}^{\dagger}\left(z_{3}\right)+\frac{z_{13}}{k-1} \partial \psi_{1}^{\dagger}\left(z_{3}\right)+\cdots\right]
$$

Therefore

$$
\lim _{z_{1} \rightarrow z_{3}}\left\langle\psi_{1}\left(z_{1}\right) \psi_{1}\left(z_{2}\right) \psi_{2}^{\dagger}\left(z_{3}\right)\right\rangle=\lim _{z_{1} \rightarrow z_{3}} \frac{\bar{c}_{1,1}(-1)^{-2 / k}}{z_{13}^{2-4 / k} z_{23}^{2-2 / k}}\left[1+\frac{2}{k} \frac{z_{13}}{z_{23}}+\cdots\right]
$$

Only the leading term contributes to the first coefficient, which is thus

$$
a_{2}=\frac{\bar{c}_{1,1}}{z_{23}^{2-4 / k}}
$$

The other coefficient is given by

$$
a_{1}=\lim _{z_{1} \rightarrow z_{3}} \frac{\partial}{\partial z_{1}}\left\{z_{13}^{2} z_{12}^{2 / k} z_{13}^{-4 / k}\left\langle\psi_{1}\left(z_{1}\right) \psi_{1}\left(z_{2}\right) \psi_{2}^{\dagger}\left(z_{3}\right)\right\rangle\right\}
$$

It is simple to check that it is equal to zero. $F\left(z_{1}\right)$ has thus a single term; the expression of the correlation function under consideration is then

$$
\left\langle\psi_{1}\left(z_{1}\right) \psi_{1}\left(z_{2}\right) \psi_{2}^{\dagger}\left(z_{3}\right)\right\rangle=\frac{\bar{c}_{1,1}}{z_{12}^{2 / k} z_{13}^{2-4 / k} z_{23}^{2-4 / k}}
$$

This is the correct three-point function. 
This computation can easily be generalized to the case where there is an arbitrary number of $\psi_{1}$ factors :

$$
\left\langle\psi_{1}\left(z_{1}\right) \cdots \psi_{1}\left(z_{n}\right) \psi_{n}^{\dagger}\left(z_{n+1}\right)\right\rangle=\prod_{1 \leq i<j \leq n} \frac{\bar{c}_{1, i}}{z_{i j}^{2 / k}} \prod_{1 \leq \ell \leq n} \frac{1}{z_{\ell, n+1}^{2-2 n / k}}
$$

Proceeding in a similar way, we can also compute the following correlator:

$$
\begin{aligned}
\left\langle\psi_{1}\left(z_{1}\right) \psi_{n}\left(z_{2}\right)\right. & \left.\psi_{n^{\prime}}\left(z_{3}\right) \psi_{n+n^{\prime}+1}^{\dagger}\left(z_{4}\right)\right\rangle=\frac{\bar{c}_{n, n^{\prime}} \bar{c}_{1, n+n^{\prime}}}{z_{12}^{2 n / k} z_{13}^{2 n^{\prime} / k} z_{23}^{2 n n^{\prime} / k}} \\
& \times \frac{1}{z_{14}^{2-2\left(n+n^{\prime}+1\right) / k} z_{24}^{2 n\left(1-\left(n+n^{\prime}+1\right) / k\right)} z_{34}^{2 n^{\prime}\left(1-\left(n+n^{\prime}+1\right) / k\right)}}
\end{aligned}
$$

We now use this last expression to extract a first result on the structure constants, by comparing the leading contribution of the limit $z_{1} \rightarrow z_{2}$ of the correlator:

$$
\begin{aligned}
\lim _{z_{1} \rightarrow z_{2}}\left\langle\psi_{1}\left(z_{1}\right) \psi_{n}\left(z_{2}\right) \psi_{n^{\prime}}\left(z_{3}\right) \psi_{n+n^{\prime}+1}^{\dagger}\left(z_{4}\right)\right\rangle \simeq \frac{\bar{c}_{1, n}}{z_{12}^{2 n / k}}\left\langle\psi_{n+1}\left(z_{2}\right) \psi_{n^{\prime}}\left(z_{3}\right) \psi_{n+n^{\prime}+1}^{\dagger}\left(z_{4}\right)\right\rangle \\
\simeq \frac{\bar{c}_{1, n} \bar{c}_{n+1, n^{\prime}}}{z_{12}^{2 n / k} z_{23}^{2 n^{\prime}(n+1) / k} z_{24}^{2(n+1)\left(1-\left(n+n^{\prime}+1\right) / k\right)} z_{34}^{2 n^{\prime}\left(1-\left(n+n^{\prime}+1\right) / k\right)}}
\end{aligned}
$$

with the same limit calculated directly from (3.19). This gives:

$$
\bar{c}_{1, n} \bar{c}_{n+1, n^{\prime}}=\bar{c}_{n, n^{\prime}} \bar{c}_{1, n+n^{\prime}}
$$

precisely the same relation found for the minimal models and thus whose solution is again exactly of the form (2.19). The observation of this factorization seems to have first been made in [11]. As already hinted at, it is typical of monomial (i.e., single term) correlators.

In order to get the complete expression for the coefficient $\bar{c}_{n, n^{\prime}}$, we simply need to evaluate $\bar{c}_{1, n}$. This can also be done by the residue method. 2 For this we need to consider the correlation function

$$
G^{\prime}=\left\langle\psi_{1}\left(z_{1}\right) \psi_{n}\left(z_{2}\right) \psi_{1}^{\dagger}\left(z_{3}\right) \psi_{n}^{\dagger}\left(z_{4}\right)\right\rangle
$$

with $n>1$. As a function of $z_{1}$,

$$
F^{\prime}\left(z_{1}\right)=z_{12}^{2 n / k} z_{13}^{-2 / k} z_{14}^{-2 n / k}\left\langle\psi_{1}\left(z_{1}\right) \psi_{n}\left(z_{2}\right) \psi_{1}^{\dagger}\left(z_{3}\right) \psi_{n}^{\dagger}\left(z_{4}\right)\right\rangle
$$

2 That was not the case for Virasoro primary-field correlators since not all $\mathcal{P}_{1}$ correlators do have a single channel. The difference here is that the $\psi_{n}$ 's have a single OPE channel. 
is a meromorphic function of $z_{1}$ with double poles at $z_{3}$ and $z_{4}$ and vanishing at infinity, so that

$$
F^{\prime}\left(z_{1}\right)=\frac{a_{2}}{z_{13}^{2}}+\frac{a_{1}}{z_{13}}+\frac{b_{2}}{z_{14}^{2}}+\frac{b_{1}}{z_{13}}
$$

A straightforward computation yields:

$$
\begin{array}{ll}
a_{2}=\frac{z_{23}^{2 n / k}}{z_{34}^{2 n / k} z_{24}^{2 n-2 n^{2} / k}} & a_{1}=-a_{2} \frac{2 n}{k} \frac{z_{24}}{z_{23} z_{34}} \\
b_{2}=\frac{c_{1, n-1}^{2}}{z_{23}^{2-2 n / k} z_{34}^{2 n / k} z_{24}^{2(n-1)-2 n^{2} / k}} & b_{1}=b_{2} \frac{2}{(k-n+1)} \frac{z_{23}}{z_{24} z_{34}}
\end{array}
$$

from which we obtain:

$$
\begin{aligned}
G^{\prime}=\left(\frac{z_{14} z_{23}}{z_{12} z_{34}}\right)^{2 n / k} & \frac{1}{z_{13}^{2-2 / k} z_{24}^{2 n-2 n^{2} / k}}\left\{1-\frac{2 n}{k} \frac{z_{13} z_{24}}{z_{23} z_{34}}\right. \\
& \left.+\bar{c}_{1, n-1}^{2} \frac{z_{13}^{2} z_{24}^{2}}{z_{23}^{2} z_{14}^{2}}\left(1+\frac{2}{k-n+1} \frac{z_{14} z_{23}}{z_{24} z_{34}}\right)\right\}
\end{aligned}
$$

To extract the value of $\bar{c}_{1, n-1}$, we compare the expression of the correlator evaluated directly in the limit $z_{12} \rightarrow 0$ and $z_{34} \rightarrow 0$, which yields

$$
\lim _{\substack{z_{1} \rightarrow z_{2} \\ z_{3} \rightarrow z_{4}}}\left\langle\psi_{1}\left(z_{1}\right) \psi_{n}\left(z_{2}\right) \psi_{1}^{\dagger}\left(z_{3}\right) \psi_{n}^{\dagger}\left(z_{4}\right)\right\rangle \simeq \frac{\bar{c}_{1, n}^{2}}{z_{12}^{2 n / k} z_{34}^{2 n / k} z_{24}^{2 n-2 n^{2} / k}}
$$

with $G^{\prime}$ given in (3.26), evaluated in the same limit. One finds that the leading terms do not match, $G^{\prime}$ being more singular. Hence, the leading coefficient in $G^{\prime}$ must cancel, which forces:

$$
\bar{c}_{1, n-1}^{2}=\frac{n(k-n+1)}{k}
$$

The substitution of this result into (2.19) leads to (3.5). Note, on the other hand, that the central charge is fixed by the subleading term of the correlator $\left\langle\psi_{1}\left(z_{1}\right) \psi_{1}\left(z_{2}\right) \psi_{1}^{\dagger}\left(z_{3}\right) \psi_{1}^{\dagger}\left(z_{4}\right)\right\rangle$.

\subsection{Some $\mathbb{Z}_{k}$ spin-field correlators and a relation between their structure constants}

A similar analysis can be applied to spin-field correlation functions. Here we use the notation $\sigma_{i}$ for the holomorphic part of $i$-th spin field in the parafermionic theory, with $\sigma_{0}=\sigma_{k}=I$. The fusion rules are

$$
\sigma_{i} \times \sigma_{j}=\sigma_{i+j}+\cdots
$$


where the dots stand for fields that are not parafermionic primary in the sense that their charge is not linked to their dimension as for the spin field, for which they are respectively

$$
h_{\ell}=\frac{\ell(k-\ell)}{2 k(k+2)} \quad \text { and } \quad q_{\ell}=\ell
$$

These fusion rules are obtained from the coset realization $\widehat{s u}(2)_{k} / \widehat{u}(1)$ [9]. Let $\tilde{c}_{i j}$ be the structure constant in front of the term which is given explicitly in (3.29), namely

$$
\left\langle\sigma_{n}\left(z_{1}\right) \sigma_{n^{\prime}}\left(z_{2}\right) \sigma_{n+n^{\prime}}^{\dagger}\left(z_{3}\right)\right\rangle=\frac{\tilde{c}_{i j}}{z_{12}^{n n^{\prime} b} z_{13}^{b n\left(k-n-n^{\prime}\right)} z_{23}^{b n^{\prime}\left(k-n-n^{\prime}\right)}}
$$

where

$$
b=\frac{1}{k(k+2)}
$$

For the general correlator of $n \sigma_{1}\left(z_{1}\right)$ fields with $\sigma_{n}^{\dagger}$, the residue method leads to the following simple expression:

$$
\left\langle\sigma_{1}\left(z_{1}\right) \cdots \sigma_{1}\left(z_{n}\right) \sigma_{n}^{\dagger}\left(z_{n+1}\right)\right\rangle=\tilde{c}_{11} \tilde{c}_{12} \cdots \tilde{c}_{1, n-1} \prod_{1 \leq i<j \leq n} \frac{1}{z_{i j}^{b}} \prod_{1 \leq i \leq n} \frac{1}{z_{i, n+1}^{(k-n) b}}
$$

This again leads to the relation (2.19) - in tilde version - for the structure constants. Obviously, this relation could be derived as previously, by considering the correlator $\left\langle\sigma_{1} \sigma_{n} \sigma_{n^{\prime}} \sigma_{n+n^{\prime}+1}^{\dagger}\right\rangle$, which leads to (2.18) whose solution is the tilde version of (2.19). It is simple to check that the explicit expression found in [4] for the $\tilde{c}_{n, n^{\prime}}$ 's

$$
\tilde{c}_{n, n^{\prime}}^{2}=\frac{\gamma(1) \gamma\left(n+n^{\prime}+1\right) \gamma(k-n+1) \gamma\left(k-n^{\prime}+1\right)}{\gamma(n+1) \gamma\left(n^{\prime}+1\right) \gamma\left(k-n-n^{\prime}+1\right) \gamma(k+1)}
$$

with

$$
\gamma(x)=\Gamma\left(\frac{x}{k+2}\right)
$$

indeed satisfies the factorization (2.19). 3 This is at the roots of the curious similarity noted in [4] between the parafermionic structure constants and the spin-field ones.

3 Note that the factorization by itself cannot lead us to the above expression: the exact value of $\tilde{c}_{1, n}$ is required and it can be obtained through the calculation of the correlator $\left\langle\sigma_{1} \sigma_{n} \sigma_{1}^{\dagger} \sigma_{n}^{\dagger}\right\rangle$. But this cannot be evaluated by means of the residue method because there are two contributing channels. In [4] it was evaluated through the coset representation. The coset approach is usually not a convenient way of computing correlation functions - (cf. [3], chap. 18); however, the simplicity of the present coset, namely $\widehat{s u}(2)_{k} / \widehat{u}(1)$, allows for a direct factorization of the correlators into a WZW piece and a free boson one. The correlator could also be calculated from the parafermionic singular conditions. This calculation will be reported elsewhere. 
3.4. $A \mathbb{Z}_{k}^{(2)}$ correlator

As already pointed out, in a parafermionic model, the conformal dimension of the parafermionic fields is an input. It is however constrained by the $\mathbb{Z}_{k}$ invariance itself, which requires $h_{\psi_{n}}=h_{\psi_{n}^{\dagger}}$ and the monodromy invariance of the correlators. These constraints are satisfied for

$$
h_{\psi_{n}}^{(\beta)}=\frac{\beta n(k-n)}{k}
$$

for any positive integer $\beta$. However, the underlying associativity conditions have to be checked anew for each value of $\beta$. The defining OPEs are given by (3.2) but with $(z-w)$ replaced by $(z-w)^{\beta}$ and $\bar{c}_{n, n^{\prime}} \rightarrow \bar{c}_{n, n^{\prime}}^{(\beta)}$. In the following, we denote the corresponding parafermionic model as $\mathbb{Z}_{k}^{(\beta)}$, with $\mathbb{Z}_{k} \equiv \mathbb{Z}_{k}^{(1)}$. The results of the associativity conditions for the case $\beta=2$ are been presented in [4] (cf. their appendix A) and the special $\mathbb{Z}_{3}^{(2)}$ model is studied in [12]. (We will report elsewhere on a detailed analysis of the $\mathbb{Z}_{k}^{(2)}$ models.)

Let us consider a sample correlator of the $\mathbb{Z}_{k}^{(2)}$ model, namely $\left\langle\psi_{1} \psi_{1} \psi_{1}^{\dagger} \psi_{1}^{\dagger}\right\rangle$, from which we construct the meromorphic function

$$
\tilde{F}\left(z_{1}\right)=z_{12}^{4 / k} z_{13}^{-4 / k} z_{14}^{-4 / k}\left\langle\psi_{1}\left(z_{1}\right) \psi_{1}\left(z_{2}\right) \psi_{1}^{\dagger}\left(z_{3}\right) \psi_{1}^{\dagger}\left(z_{4}\right)\right\rangle
$$

It has poles or order 4 at $z_{3}$ and $z_{4}$ and behaves as $1 / z_{1}^{4}$ as $z_{1} \rightarrow \infty$, meaning that there is no analytic piece. The computation of the two principal parts requires thus the knowledge of the first three subleading terms in the OPE $\psi_{1}(z) \psi_{1}^{\dagger}(w)$. That makes the computation much more involved than in the $\beta=1$ case. However, there is a simple trick that allows us to avoid going so deeply inside the conformal block. Since $F \sim 1 / z_{1}^{4}$, we can reduce the order of the two poles by two, at the price of adding a constant term, by multiplying $\tilde{F}\left(z_{1}\right)$ by $z_{13}^{2} z_{14}^{2}$. The transformed meromorphic function reads thus

$$
\tilde{F}^{\prime}\left(z_{1}\right)=z_{13}^{2} z_{14}^{2} \tilde{F}\left(z_{1}\right)=\frac{a_{2}}{z_{13}^{2}}+\frac{a_{1}}{z_{13}}+\frac{b_{2}}{z_{14}^{2}}+\frac{b_{1}}{z_{14}}+c_{0}
$$

For this computation we require the knowledge of only the first subleading term in the OPE $\psi_{1}(z) \psi_{1}^{\dagger}(w)$. A direct but rather long calculation gives the following expression for the correlator:

$$
\begin{aligned}
& \frac{1}{\left(z_{12} z_{34}\right)^{4 / k}\left(z_{13} z_{14} z_{23} z_{24}\right)^{2-4 / k}}\left\{\left(\bar{c}_{11}^{(2)}\right)^{2}+\frac{z_{34}^{2}}{z_{23} z_{24}}-\frac{4}{k} \frac{z_{12}^{2} z_{34}^{2}}{z_{14} z_{13} z_{23} z_{24}}\right. \\
& \left.+\frac{z_{34} z_{24}}{z_{14}^{2} z_{23}^{2}}\left(z_{12} z_{14}-z_{13} z_{24}\right)+\frac{z_{34} z_{23}}{z_{24}^{2} z_{13}^{2}}\left(z_{14} z_{23}-z_{12} z_{13}\right)\right\}
\end{aligned}
$$


If we compare this expression in the limit $z_{1} \rightarrow z_{3}$ with the correlator evaluated directly in this same limit, we find that for $c$ expressed in terms of a parameter $\lambda$ as

$$
c=\frac{4(k-1) \lambda(k+\lambda-1)}{(k+2 \lambda) k+2 \lambda-2)}
$$

the structure constant $\bar{c}_{11}^{(2)}$ becomes

$$
\left(\bar{c}_{11}^{(2)}\right)^{2}=\frac{2(k-1)(\lambda+1)(k+\lambda-2)}{k \lambda(k+\lambda-1)}
$$

in agreement with [12]. To check that the $\mathbb{Z}_{k}^{(2)}$ central charge is unconstrained, we need to compare the subleading terms.

Here again the constants $\bar{c}_{n, n^{\prime}}^{(2)}$ have the factorization property (2.19), so that all constants can be expressed in terms of $\bar{c}_{1, n}^{(2)}$, whose determination requires the evaluation of $\left\langle\psi_{1} \psi_{n} \psi_{1}^{\dagger} \psi_{n}^{\dagger}\right\rangle$. Again the calculation can be reduced to the evaluation of poles of order two. The expression of all the structure constants $\bar{c}_{n, n^{\prime}}^{(2)}$ are given in [12]. Curiously, $\bar{c}_{n, n^{\prime}}^{(2)}$ has another factorization, namely as

$$
\bar{c}_{n, n^{\prime}}^{(2)}=\bar{c}_{n, n^{\prime}} d_{n, n^{\prime}}(\lambda), \quad \text { with } \quad d_{n, n^{\prime}}(1)=\bar{c}_{n, n^{\prime}} \equiv \bar{c}_{n, n^{\prime}}^{(1)}
$$

whose origin appears somewhat mysterious.

The structure constants for the models $\mathbb{Z}_{k}^{(\beta>2)}$ can be computed in the same manner. Yet there exits no results concerning these theories.

Coming back to methodological aspects, with the computation presented in this subsection, we wanted to emphasis that the evaluation of the principal part can be substantially simplified by an appropriate modification of the integer powers of the prefactor multiplying the correlator. Here, poles of order 4 have been transformed into poles of order 2. This is not a purely technical issue. The determination of higher subleading terms in the OPE $\psi^{\dagger}$ requires the knowledge of the chiral algebra underlying the $\mathbb{Z}_{k}^{(2)}$ models. More precisely, the required information can be extracted from various associativity constraints but it is clear that knowing at least the gross features of the underlying chiral algebra is useful. For the $\mathbb{Z}_{k}^{(1)}$ model, it is the $W A_{k-1}$ algebra. However, the chiral algebra of the $\mathbb{Z}_{k}^{(2)}$ models is not known. 


\section{Conclusion}

We have thus exposed, in its full generality, a method for calculating special CFT correlators based an a direct application of the residue calculus. Let us first summarize the method and restate the limits of its applicability.

We consider the correlator $\left\langle A(z) \prod_{i} B_{i}\left(z_{i}\right)\right\rangle$ as a function of $z$. The first step is to transform it into a meromorphic function of $z$. This is already guaranteed if the OPEs $A(z) B_{i}\left(z_{i}\right)$ are Laurent series, which requires that the conformal dimensions of all the fields that appear in the singular terms of the OPE $A(z) B_{i}\left(z_{i}\right)$, as well as $A$ and $B_{i}$, differ by integers. This situation pertains to correlators of chiral-algebra generators (in which case all the fields have integer dimension). However, when $A$ is not a symmetry generator, the above condition is rarely verified. Generically, the OPE $A(z) B_{i}\left(z_{i}\right)$ contains a number of channels associated to primary fields whose dimensions do not differ by integers. In such a case, one can still construct a meromorphic function if either there is a single channel, or more generally, if a single channel, say $C_{i}$, contributes to the correlation function. This, however, does not ensure that $h_{A}+h_{B_{i}}-h_{C_{i}}$ is integer, i.e., that the OPE projected onto the $C_{i}$ channel is a Laurent series. But the cure at this point is simple: one multiplies the OPE by a fractional power of $z-z_{i}$ suitably chosen to eliminate the algebraic singularity, leaving thus pole-type singular terms. In other words, when the effective single-channel requirement is satisfied, the correlator can be transformed into a meromorphic function of

$z$ by multiplying it by an appropriate factor $\prod_{i}\left(z-z_{i}\right)^{d_{i}}$. This meromorphic function is then expanded in partial fractions, that is, as the sum of the principal parts at the different $z_{i}$, including possibly the principal part at infinity.

This method is certainly not new and we try in appendix B to trace it back in the literature. However, its explicit spelling out as well as the identification of its inherent limitations appear to be new. The method has been illustrated here with various examples, including some correlators that do not involve conserved currents. As a practical application, we have worked out a detailed derivation of the parafermionic structure constants.

\section{Appendix A. A three-point function computed from the infinite series}

In this appendix, we consider the calculation of the three-point function $\left\langle T\left(z_{1}\right) T\left(z_{2}\right) T\left(z_{3}\right)\right\rangle$ using the brute force infinite-series method, where one OPE is replaced by its full infinite 
series and show how this series can be summed exactly to reproduce the simple three-point function expression.

The long road computation will be compared to the few step computations that results from the application of the conformal Ward identity:

$$
\begin{aligned}
& \left\langle T\left(z_{1}\right) T\left(z_{2}\right) T\left(z_{3}\right)\right\rangle=\left\langle\overparen{T\left(z_{1}\right) T}\left(z_{2}\right) T\left(z_{3}\right)\right\rangle+\left\langle T\left(z_{2}\right) \stackrel{T\left(z_{1}\right) T}{T}\left(z_{3}\right)\right\rangle \\
& =\left\langle\left(\frac{c / 2}{z_{12}^{4}}+\frac{2 T\left(z_{2}\right)}{z_{12}^{2}}+\frac{\partial T\left(z_{2}\right)}{z_{12}}\right) T\left(z_{3}\right)\right\rangle+\left\langle T\left(z_{2}\right)\left(\frac{c / 2}{z_{13}^{4}}+\frac{2 T\left(z_{3}\right)}{z_{13}^{2}}+\frac{\partial T\left(z_{3}\right)}{z_{13}}\right)\right\rangle \\
& \quad=\frac{c}{z_{12}^{2} z_{23}^{4}}-\frac{2 c}{z_{12} z_{23}^{5}}+\frac{c}{z_{13}^{2} z_{23}^{4}}+\frac{2 c}{z_{13} z_{23}^{5}}=\frac{c}{z_{12}^{2} z_{13}^{2} z_{23}^{2}}
\end{aligned}
$$

Let us now turn to the expression that results from the replacement of the OPE $T\left(z_{1}\right) T\left(z_{2}\right)$ by its infinite series:

$$
\begin{aligned}
T\left(z_{1}\right) T\left(z_{2}\right) & =\overparen{T\left(z_{1}\right) T}\left(z_{2}\right)+\left(T\left(z_{1}\right) T\left(z_{2}\right)\right) \\
& =\left(\frac{c / 2}{z_{12}^{4}}+\frac{2 T\left(z_{2}\right)}{z_{12}^{2}}+\frac{\partial T\left(z_{2}\right)}{z_{12}}\right)+\sum_{n=0}^{\infty} \frac{z_{21}^{n}}{n !}\left(T T^{(n)}\right)\left(z_{1}\right)
\end{aligned}
$$

where $T^{(n)}=\partial^{n} T$ and $\left(T\left(z_{1}\right) T\left(z_{2}\right)\right)$ stands for the normal ordering of the product $T\left(z_{1}\right) T\left(z_{2}\right)$, defined as

$$
(A B)(z)=\frac{1}{2 \pi i} \oint \frac{d x}{x-z} A(x) B(z)
$$

The substitution of (A.2) into the three-point function yields

$$
\left\langle T\left(z_{1}\right) T\left(z_{2}\right) T\left(z_{3}\right)\right\rangle=\frac{c}{z_{12}^{2} z_{23}^{4}}-\frac{2 c}{z_{12} z_{23}^{5}}+\sum_{n=0}^{\infty} \frac{z_{21}^{n}}{n !}\left\langle\left(T T^{(n)}\right)\left(z_{1}\right) T\left(z_{3}\right)\right\rangle
$$

In the last three-point function, we have to find the term proportional to $c z_{13}^{-6-n}$ which is the only contributing one; it is obtained by standard methods (see e.g., [3]): in

$$
\left.\overparen{T\left(z_{3}\right)(T} T^{(n)}\right)\left(z_{1}\right)=\frac{1}{2 \pi i} \oint \frac{d x}{x-z_{1}}\left\{\left(\overleftrightarrow{T\left(z_{3}\right) T}(x)\right) T^{(n)}\left(z_{1}\right)+T(x)\left(\overparen{T\left(z_{3}\right) T^{(n)}}\left(z_{1}\right)\right)\right\}
$$

only the first piece contributes to $c z_{31}^{-6-n}$ and its different contributions add up $(6+n)(3+$ $n) ! / 12$. Therefore, we have

$$
\left\langle\left(T\left(z_{1}\right) T\left(z_{2}\right)\right) T\left(z_{3}\right)\right\rangle=\frac{c}{12} \sum_{n=0}^{\infty} \frac{(3+n) !(6+n)}{n !} \frac{z_{12}^{n}}{z_{13}^{n+6}}
$$


The infinite series can be summed as follows:

$$
\begin{aligned}
& \sum_{n=0}^{\infty}(3+n)(2+n)(1+n)(6+n) \frac{z_{12}^{n}}{z_{13}^{n}}=\frac{1}{z_{12}^{5}} \partial_{2} z_{12}^{6} \partial_{2}^{3} \sum_{n=0}^{\infty} \frac{z_{12}^{n+3}}{z_{13}^{n}} \\
& \quad=\frac{1}{z_{12}^{5}} \partial_{2} z_{12}^{6} \partial_{2}^{3}\left(\frac{z_{12}^{3}}{1-z_{12} / z_{13}}\right)=\frac{1}{z_{12}^{5}} \partial_{2} z_{12}^{6} \partial_{2}^{3}\left(\frac{z_{12}^{3} z_{13}}{z_{23}}\right) \\
& \quad=\frac{1}{z_{12}^{5}} \partial_{2} z_{12}^{6}\left(\frac{-6 z_{13}^{3}}{z_{23}^{4}}\right)=\frac{36 z_{13}^{3}}{z_{23}^{4}}+\frac{24 z_{13}^{3} z_{12}}{z_{23}^{5}}
\end{aligned}
$$

so that

$$
\left\langle\left(T\left(z_{1}\right) T\left(z_{2}\right)\right) T\left(z_{3}\right)\right\rangle=\frac{c}{z_{13}^{2} z_{23}^{5}}\left(3 z_{23}+2 z_{12}\right)=\frac{c}{z_{13}^{2} z_{23}^{5}}\left(z_{23}+2 z_{13}\right)
$$

The substitution of this expression into (A.4) reproduces (A.1).

\section{Appendix B. Correlators as meromorphic functions through the literature}

As applied to correlators containing symmetry generators, the residue method could be traced back to the pioneer work of Belavin, Polyakov and Zamolodchikov [1]. These authors explicitly consider the correlator $\left\langle T(z) \prod_{i} \phi_{i}\left(z_{i}\right)\right\rangle$ as a meromorphic function of $z$ with poles at $z_{i}$ whose residues are fixed by the conformal properties of the field $\phi_{i}\left(z_{i}\right)$ (cf. the discussion after their eqs (2.8) and (3.3)). The method is also used in [2]. In this seminal paper, Zamoldchikov has launched the exploration of extended conformal algebras through the study of the associativity conditions of a number of cases containing a single extra symmetry generator. Correlation functions are computed by considering only the singular terms in the OPEs. There is again an explicit reference to the residue calculus (cf. eqs (2.1)-(2.4)) that suggests an underlying complex-analysis interpretation of the exposed computations. But note that such an approach is not mandatory since the calculation method could be justified by means of the Ward identities associated to these extra conserved currents.

Meromorphicity is the central theme of Goddard's proposed formalization of conformal field theory [13]. However, the meromorphic point of view here is not implemented at the level of computing correlation functions. In this context, meromorphicity is used to establish locality which in turn becomes the corner-stone property for the construction of the conformal field theory. This formal procedure has been much developed in a sequel work with Gaberdiel [14]. Here the emphasis is placed on the reconstruction the whole 
theory out of meromrophic amplitudes, via an approach inspired by the early works in dual models where the space of states was originally built out of the conjectured dual amplitudes. In this very paper however, the residue method is explicitly invoked for the calculation of some correlators involving symmetry generators. In that regard, they present some results of Frenkel and Zhu [15], which have devised a nice combinatorial method for handling such correlators (involving an arbitrary number of either $T$ factors or affine Lie algebra generators - summarized in sections 5(b) and 5(c) of [14]). But again, the residue method is not systematized and its applications are restricted to the elimination of conserved currents in correlation functions.

It is in the context of a non-meromorphic theory that the partial fraction expansion technique has been mentioned in the most explicit way, namely as a natural tool for evaluating the parafermionic correlator $\left\langle\psi_{1} \cdots \psi_{1} \psi_{1}^{\dagger} \cdots \psi_{1}^{\dagger}\right\rangle[4]$ (cf. the discussion between eqs (3.9)-(3.12)). In this paper, the authors also give the value of the parafermionic structure constants. Even though there are no indication concerning the way these have been computed, it is natural to guess that their calculations have been done roughly along the lines presented here.

A variant of the partial fraction expansion method has been used explicitly in [11] to work out in detail the associativity conditions of the $\mathbb{Z}^{(2)}$ models (cf. their section $3 \mathrm{~B}$ ). The approach used there is superficially a little more complicated that the one presented here in that the four-point correlation functions are transformed into meromorphic functions (actually, into polynomials) of the cross ratio instead of functions of the position of one field. This procedure prevents an immediate generalization to higher-point functions.

As applied to correlators that do not contain symmetry generators, we found afterwards a single reference to the residue method: this is in appendix $\mathrm{E}$ of [1] (cf. eqs (E.14)-(E.17)). It is used there to calculate the Ising correlator $\left\langle\psi(z) \sigma\left(z_{1}\right) \cdots \mu\left(z_{2 M}\right)\right\rangle$ where $\psi$ is the Ising fermion and $\sigma$ and $\mu$ are respectively the spin and disorder fields. The correlator is transformed into a meromrophic function of $z$ as follows:

$$
F(z)=\left(\prod_{i=1}^{2 M}\left(z-z_{i}\right)^{1 / 2}\right)\left\langle\psi(z) \sigma\left(z_{1}\right) \cdots \mu\left(z_{2 M}\right\rangle\right.
$$

Actually, all singularities in $z$ are removed by this transformation since

$$
\psi(z) \sigma(w) \sim \frac{\mu(w)}{(z-w)^{1 / 2}} \quad \psi(z) \mu(w) \sim \frac{\sigma(w)}{(z-w)^{1 / 2}}
$$


Observe that these OPEs have a single channel. $F(z)$ is thus an analytic function. The form of this analytic function is determined by its behavior at infinity. Since $F(z) \sim z^{M-1}$ as $z \rightarrow \infty$, it is necessarily a polynomial of order $M-1$, which can be written as

$$
F(z)=\sum_{k=1}^{M-1}\left(z-z_{2 M}\right)^{k} g_{k}\left(z_{i}\right)
$$

(disregarding the dependence upon the anti-holomorphic variables). are determined by enforcing the correlator to be a solution of the singular-vector differential equation of the free fermion $\left(=\phi_{21}\right)$. Quite interestingly, the coefficient $g_{0}$ gives the value of the correlation function without the fermion.

More recently, Dotsenko [16] has devised a nice way of handling the parafermionic computations by a method which is close in spirit to the computation of [1] just described, hence to the partial fraction expansion. Applied to four-point functions with three points fixed at the special values $0,1, \infty$, the idea is to factor out the branching or poles singularities of the resulting $z$ function and then determine the remaining polynomial in $z$ that completes the correlator by considering successively the correlator in the limits where $z$ approaches the three fixed points. A detailed application of this method is presented in the following appendix.

\section{Appendix C. The Dotsenko's method to test associativity}

We will illustrate the method initiated by Dotsenko by reconsidering the $\mathbb{Z}_{k}$ parafermionic correlator $\left\langle\psi_{1}\left(z_{1}\right) \psi_{n}\left(z_{2}\right) \psi_{1}^{\dagger}\left(z_{3}\right) \psi_{n}^{\dagger}\left(z_{4}\right)\right\rangle$. We fix three points at the standard values 0,1 and $\infty$ and use the following convention for a 'prime correlator':

$$
\langle X A(\infty)\rangle^{\prime} \equiv \lim _{z_{n} \rightarrow \infty} z_{n}^{2 h_{A}}\left\langle X A\left(z_{n}\right)\right\rangle
$$

In particular, we have

$$
\left\langle\psi_{n}(z) \psi_{n}^{\dagger}(\infty)\right\rangle^{\prime}=1, \quad\left\langle\psi_{n}(z) \psi_{n}(1) \psi_{n+n^{\prime}}^{\dagger}(\infty)\right\rangle^{\prime}=\frac{\bar{c}_{n, n^{\prime}}}{(z-1)^{2 n n^{\prime} / k}}
$$

4 The choice of the expansion variable, here $z-z_{2 M}$, is conventional; another $z_{j}$ could have be chosen instead of $z_{2 M}$ and the power series could also have been written in powers of $z$ simply. Writing the expansion in terms of a difference between two variables has the advantage of taking care of translation invariance. Note that there is a misprint on the value of the upper limit of the sum in (E.16) of [1]. 
We consider thus $\left\langle\psi_{1}(0) \psi_{n}(z) \psi_{1}^{\dagger}(1) \psi_{n}^{\dagger}(\infty)\right\rangle$. Regarded as a function of $z$, this has singularities at 0 and 1 , that is

$$
\mathcal{G}(z) \equiv\left\langle\psi_{1}(0) \psi_{n}(z) \psi_{1}^{\dagger}(1) \psi_{n}^{\dagger}(\infty)\right\rangle^{\prime}=\frac{P_{n}(z)}{z^{2 n / k}(z-1)^{2-2 n / k}}
$$

(we do not care about the phases which all cancel at the end). $P_{n}$ is a polynomial of order $n$. In other words, by multiplying the correlator by the prefactor $z^{2 n / k}(z-1)^{2-2 n / k}$, we transform it into a meromorphic function, which turns out to be analytic.

The order of $P_{n}$ is fixed by considering the limit where $z \rightarrow \infty$, where we readily see that $\mathcal{G}(\infty)=1$, i.e.,

$$
\lim _{z \rightarrow \infty} \mathcal{G} \sim \lim _{z \rightarrow \infty}\left\langle\psi_{1}(0) \psi_{1}^{\dagger}(1)\right\rangle\left\langle\psi_{n}(z) \psi_{n}^{\dagger}(\infty)\right\rangle^{\prime}=1
$$

This implies that $n=2$ and, in addition, that the coefficient of the term $z^{2}$ is 1 , that is

$$
P_{2}(z)=a_{0}+a_{1} z+z^{2}
$$

Thus, in order to completely determine the correlator, we only have to fix these two constants.

Consider first the limit where $z \rightarrow 0$, keeping track of the first subleading term. The correlator $\mathcal{G}(z)$ becomes then

$$
\begin{aligned}
\lim _{z \rightarrow 0} \mathcal{G}(z) & =\frac{\bar{c}_{1, n}}{z^{2 n / k}}\left\langle\psi_{n+1}(z) \psi_{1}^{\dagger}(1) \psi_{n}^{\dagger}(\infty)\right\rangle-\frac{z}{n+1} \frac{\bar{c}_{1, n}}{z^{2 n / k}}\left\langle\partial \psi_{n+1}(z) \psi_{1}^{\dagger}(1) \psi_{n}^{\dagger}(\infty)\right\rangle \\
& =\frac{\bar{c}_{1, n}^{2}}{z^{2 n / k}(z-1)^{2-2(n+1) / k}}\left\{1+\frac{2(k-n-1) z}{k(n+1)(z-1)}\right\} \\
& \simeq \frac{\bar{c}_{1, n}^{2}}{z^{2 n / k}}\left\{1+\frac{2 k(k-n-1) z}{k(n+1)}\right\}
\end{aligned}
$$

This is to be compared with the expansion of the rhs of (C.3):

$$
\lim _{z \rightarrow 0}\left\{\frac{a_{0}+a_{1} z+z^{2}}{z^{2 n / k}(z-1)^{2-2 n / k}}\right\} \simeq \frac{1}{z^{2 n / k}}\left[a_{0}+z\left(a_{1}+a_{0} \frac{2(k-n)}{k}\right)\right]
$$

This yields

$$
a_{0}=\bar{c}_{1, n}^{2}, \quad a_{1}=-\frac{2 a_{0}}{n+1}
$$


Next we consider the limit $z \rightarrow 1$. Here it will suffices to keep only the leading term. We have thus

$$
\lim _{z \rightarrow 1} \mathcal{G}(z) \simeq \frac{\bar{c}_{1, n-1}}{(z-1)^{2-2 n / k}}\left\langle\psi_{1}(0) \psi_{k-1}^{\dagger}(1) \psi_{n}^{\dagger}(\infty)\right\rangle \simeq \frac{\bar{c}_{1, n-1}^{2}}{(z-1)^{2-2 n / k}}
$$

which is to be compared to

$$
\lim _{z \rightarrow 1}\left\{\frac{a_{0}+a_{1} z+z^{2}}{z^{2 n / k}(z-1)^{2-2 n / k}}\right\} \simeq \frac{a_{0}+a_{1}+1}{(z-1)^{2-2 n / k}}
$$

That forces

$$
a_{0}+a_{1}+1=\bar{c}_{1, n-1}^{2}
$$

which together with (C.8) yields the recursion relation

$$
\bar{c}_{1, n-1}^{2}=\bar{c}_{1, n}^{2}\left(\frac{n-1}{n+1}\right)+1
$$

This relation can be solved without knowing the explicit value of $\bar{c}_{11}$ but simply by enforcing the condition

$$
\bar{c}_{1, n}=\bar{c}_{1, k-n-1}
$$

This readily implies

$$
\bar{c}_{1, k-n}^{2}=\bar{c}_{1, k-n-1}^{2}\left(\frac{n-1}{n+1}\right)+1
$$

On the other hand, replacing $n$ by $k-n$ in (C.11) yields

$$
\bar{c}_{1, k-n-1}^{2}=\bar{c}_{1, k-n}^{2}\left(\frac{k-n+1}{k-n-1}\right)+1
$$

Eliminating $\bar{c}_{1, k-n}^{2}$ from the last two equations lead to an algebraic relation for $\bar{c}_{1, k-n-1}^{2}$ which gives

$$
\bar{c}_{1, k-n-1}^{2}=\bar{c}_{1, n}^{2}=\frac{(n+1)(k-n)}{k}
$$

and we recover the expression of $\bar{c}_{1, n}$ obtained previously in (3.28).

At first sight, it seems that the applicability of this method depends critically upon the fact that the field evaluated at $z$ is the conjugate of the one at infinity. In that case, the other two fields are conjugate of each other, which ensures that the fractional powers of $z$ cancel in the denominator as $z \rightarrow \infty$. That certainly ensures the polynomial character of $P_{n}$ 
as in the large $z$ behavior $\psi_{n}(z)$ is simply projected onto $\psi_{n}^{\dagger}(\infty)$ and $\left\langle\psi_{n}(z) \psi_{n}^{\dagger}(\infty)\right\rangle^{\prime}=1$. But consider instead the correlator

$$
\left\langle\psi_{1}(0) \psi_{n}(z) \psi_{n}^{\dagger}(1) \psi_{1}^{\dagger}(\infty)\right\rangle^{\prime}=\frac{Q(z)}{z^{2 n / k}(z-1)^{2 n-2 n^{2} / k}}
$$

It is not clear at once that $Q(z)$ is polynomial here. The point however is that the large $z$ limit of $\langle A(0) B(z) C(1) D(\infty)\rangle^{\prime}$ is actually given by $z^{h_{B}+h_{D}-h_{E}} / z^{2 h_{B}}$ where $E$ is the single contributing field appearing in the OPE of $B$ and $D$. Indeed, by considering at first the limit $z_{4} \rightarrow \infty$, we wash out the contribution $z_{24}^{h_{B}+h_{D}-h_{E}}$ that needs to be reinserted at this point in order to get the right large $z$ behavior. The term $z^{-2 h_{B}}$ simply corresponds to the large $z$ behavior of the $B$ field. Returning to our problem, we need to compare the large $z$ limit of the rhs of (C.16) with $z^{2-2 n+2 n^{2} / k-2 n / k} \sim z^{2}$ which shows that $Q(z)$ is indeed a polynomial of degree 2 .

It should be clear from this example that the Dotsenko's method has the same intrinsic limitations as the partial fraction expansion described in the main part of the article. In particular, it generically applies to correlators that have a single contributing channel.

\section{Acknowledgment}

We thank V.I. Dotsenko for very stimulating discussions that have triggered our interest for the subject of this work. We would also like to thank M. Gaberdiel (who pointed out to us [14] and [15]), Y. Saint-Aubin and G. Watts for useful comments and J.-F. Carrier and D. Huard for exploratory calculations related to this work. We are also grateful to P. Furlan for pointing out [11]. We acknowledge the financial support of NSERC and FCAR.

\section{REFERENCES}

1. A.A. Belavin, A.M. Polyakov and A.B. Zamolodchikov, Nucl. Phys. B241 (1984) 333.

2. A.L. Zamolodchikov, Theo. Math. Phys. 63 (1985) 1205.

3. P. Di Francesco, P. Mathieu and D. Sénéchal, Conformal Field theory, Springer Verlag, 1997.

4. A.B. Zamolodchikov and V.A. Fateev, Sov. Phys. JETP 43 (1985) 215.

5. Vl.S. Dotsenko and V.A. Fateev, Nucl. Phys. B235 (1984) 312.

6. Vl.S. Dotsenko and V.A. Fateev, Nucl. Phys. B251 (1985) 691.

7. Vl.S. Dotsenko and V.A. Fateev, Phys. Lett. 154B (1985) 291.

8. A.B. Zamolodchikov and V.A. Fateev, Sov. Phys. JETP 63 (1986) 913. 
9. D. Gepner and Z. Qiu, Nucl. Phys. 285 (1987) 423.

10. P. Jacob and P. Mathieu, Nucl. Phys. B 587 (2000) 514.

11. P. Furlan, R.R. Paunov and I.V. Todorov, Fortschr. Phys. 40 (1992) 211.

12. A.B. Zamolodchikov and V.A. Fateev. Theor. Math. Phys. 71 (1987) 163.

13. P. Goddard, Meromorphic conformal field theory, in Infinite dimensional Lie algebras and groups, ed. V. Kac, World Scientific (1989) 556.

14. M. Gaberdiel and P. Goddard, Commun.Math.Phys. 209 (2000) 549.

15. I.B. Frenkel and Y. Zhu, Duke Math. J., 66 (1992) 123.

16. Vl.S. Dostsenko, lectures at LPTHE (unpublished) and informal lecture at the workshop Quantum integrability, CRM, May 2000; Vl.S.Dotsenko, J.L.Jacobsen and M.Picco, Parafermionic Theories, to be published. 